Research Paper

\title{
mTOR/autophagy pathway in the hippocampus of rats suffering intermittent hypoxia preconditioning and global cerebral ischemia-reperfusion
}

\author{
Ya-Ning Zhao ${ }^{1}$, Xiang-Fei Guo ${ }^{1}$, Jian-Min $\mathrm{Li}^{2}$, Chang-Xiang Chen ${ }^{1}$, Shu-Xing $\mathrm{Li}^{1}$, \\ Cheng-Jing $\mathrm{Xu}^{2}$ \\ ${ }^{1}$ Nursing and Rehabilitation College, North China University of Science and Technology, 063000, China \\ ${ }^{2}$ The Neurosurgery of Affiliated Hospital, North China University of Science and Technology, 063000, China \\ Correspondence to: Jian-Min Li, email: lijianmjm7@163.com \\ Keywords: OSAHS-patterned hypoxia, cerebral ischemia-reperfusion, mTOR, autophagy \\ Received: November 04, 2016 \\ Accepted: December 12, 2016 \\ Published: February 03, 2017
}

\section{ABSTRACT}

We explored the role of $\mathrm{mTOR} /$ autophagy pathway in the aggravation of cerebral ischemia-reperfusion nerve injury caused by intermittent hypoxia. Eighty male wistar rats were divided into four groups by the random number method: sham operation group (SO group, $n=20$ ), cerebral ischemia-reperfusion group ( $I / R$ group, $n=20$ ), intermittent hypoxia and cerebral ischemia-reperfusion group ( $I H+I / R$ group, $\mathbf{n = 2 0}$ ), intermittent hypoxia and cerebral ischemia-reperfusion group plus mTOR inhibitor group (inhibitor group, $n=20$ ). The results showed that compared with the so group, HE staining showed structural damage of neurons at each time point, the immunohistochemical assay showed an increasing number of mTOR and beclin1 immune-positive cells $(P<0.05)$ and $R T-P C R$ showed enhanced expression of $m$ TOR and beclin 1 protein in the $I / R$ group $(P<0.05)$. Compared with the $I / R$ group, $H E$ staining showed exacerbating structural damage of neurons at each time point, the immunohistochemical assay showed an increasing number of $m$ TOR and beclin 1 immune-positive cells $(P<0.05)$ and $R T-P C R$ showed enhanced expression of $m$ TOR and beclin 1 protein in the $I H+I / R$ group $(P<0.05)$. Compared with the $I H+I / R$ group, HE staining showed remissive structural damage of neurons at each time point, the immunohistochemical assay showed a decreasing number of mTOR immune-positive cells and a rising number of beclin 1immune-positive cells $(P<0.05)$ and $R T-P C R$ showed weakened expression of mTOR protein and enhanced expression of beclin 1 protein in the inhibitor group $(P<0.05)$. Thence, the present study indicated that intermittent hypoxia preconditioning can aggravate the nerve injury of the global cerebral ischemia-reperfusion model, and the mechanism is associated with the activation of $\mathrm{mTOR} /$ autophagy pathway.

\section{INTRODUCTION}

Obstructive sleep apnea-hypopnea syndrome (OSAHS) is an independent risk factor of cerebral arterial thrombosis, which can aggravate the cerebral injury caused by cerebral arterial thrombosis [1-2]. Autophagy, which widely exists in cerebrovascular diseases such as cerebral arterial thrombosis, exerts significant effects on the pathological process of cerebral arterial thrombosis via regulating apoptosis of nerve cells [3-4]. Mammalian target of rapamycin (mTOR) is an evolutionarily conserved serine/threonine protein kinase, which can be stimulated by various factors such as growth factors, nutriments, etc., and then phosphorylate downstream target proteins to engage in genetic transcription and protein expression and affect autophagy [5-6]. Studies have shown that rapamycin can lessen hypoxic-ischemic brain damage of new-born rats, in which mTOR plays an important part [7]. ROS, oxidative stress, and inflammation responses, etc., aggravate OSAHS complicated with cerebral arterial thrombosis, which all can result in autophagy of nerve cells in a direct or indirect manner. Therefore, we speculate that 
mTOR/autophagy-associated pathways play an important role in the pathological process of the aggravation of cerebral ischemia nerve injury caused by intermittent hypoxia. In this study, we used rats with intermittent hypoxia preconditioning to prepare global cerebral ischemia-reperfusion models, observed the expression of mTOR/autophagy pathway and the loss and changes of nerve cells in the rat hippocampus and investigated its role in the aggravation of cerebral ischemia-reperfusion neuron injury caused by intermittent hypoxia, so as to provide experimental basis for the prevention and cure of OSAHS complicated with ischemic cerebrovascular diseases.

\section{RESULTS}

\section{HE staining}

The hippocampus nerve cells of the SO group showed orderly arrangement, normal structure, clear nuclei and distinct nucleoli. The I/R group showed many swollen neurons with loosen structure and deep-dyed and pyknotic nuclei, with a part of neurons completely losing their nuclei, forming a vacuolus-like structure, and showed a markedly decreased density of survival neurons at each time point $(6 \mathrm{~h}$ and $24 \mathrm{~h})(\mathrm{P}<0.05)$. The $\mathrm{IH}+\mathrm{I} / \mathrm{R}$ group showed even more seriously damaged structure of neurons, more denatured and necrotic neurons with loosen structure, deep-dyed and pyknotic nuclei in neurons with a part of neurons completely losing their nuclei, forming a vacuoluslike structure, and showed a further diminished density of survival neurons at each time point (6h and 24h) $(\mathrm{P}<0.05)$. Compared with the $\mathrm{IH}+\mathrm{I} / \mathrm{R}$ group, the inhibitor group showed a lower degree of structural damage of neurons as well as an increased survival density of neurons at each time point (6h and 24h) $(\mathrm{P}<0.05)$. (See Table 1 and Figure 1)

\section{Immunohistochemical staining}

\section{mTOR}

mTOR manifests as a brown color after immunohistochemical staining, and it is located in cytoplasm and primarily expressed in neurons. mTOR immune-positive cells were rarely seen in the SO group; compared with the SO group, the I/R group showed an increased number of mTOR immune-positive cells at each time point $(\mathrm{P}<0.05)$; compared with the I/R group, the $\mathrm{IH}+\mathrm{I} / \mathrm{R}$ group also showed an increased number of mTOR immune-positive cells at each time point $(\mathrm{P}<0.05)$; compared with the $\mathrm{IH}+\mathrm{I} / \mathrm{R}$ group, the inhibitor group showed a decreased number of mTOR immune-positive cells at each time point $(\mathrm{P}<0.05)$. (See Table 2 and Figure 2)

\section{Beclin1}

Beclin1 manifests as a brown color after immunohistochemical staining, and it is located in cytoplasm and primarily expresses in neurons. Beclin1 immune-positive cells were rarely seen in the SO group; compared with the $\mathrm{SO}$ group, the I/R group showed an increased number of beclin1 immune-positive cells at each time point $(\mathrm{P}<0.05)$; compared with the I/R group, each of the OSAHS hypoxia groups showed an increased number of beclin 1 immunepositive cells at each time point $(\mathrm{P}<0.05)$; compared with the $\mathrm{IH}+\mathrm{I} / \mathrm{R}$ group, the inhibitor group also showed an increased number of beclin 1 immune-positive cells at each time point $(\mathrm{P}<0.05)$. (See Table 2 and Figure 2)

\section{RT-PCR}

\section{mTOR}

Compared with the SO group, the I/R group showed remarkably enhanced expression of $\mathrm{mTOR}$ protein at each time point ( $6 \mathrm{~h}$ and $24 \mathrm{~h})(\mathrm{P}<0.05)$; compared with the I/R group, the IH $+\mathrm{I} / \mathrm{R}$ group showed enhanced expression of mTOR protein at each time point $(6 \mathrm{~h}$ and $24 \mathrm{~h})(\mathrm{P}<0.05)$; compared with the $\mathrm{IH}+\mathrm{I} / \mathrm{R}$ group, the inhibitor group showed down-regulated expression of mTOR protein at each time point $(6 \mathrm{~h}$ and $24 \mathrm{~h})(\mathrm{P}<0.05)$. (See Table 3 )

\section{Beclin1}

Compared with the SO group, the I/R group showed remarkably enhanced expression of beclin 1 protein at each time point ( $6 \mathrm{~h}$ and $24 \mathrm{~h})(\mathrm{P}<0.05)$; compared with the $\mathrm{I} / \mathrm{R}$ group, each of the OSAHS hypoxia groups showed enhanced expression of beclin1 protein at each time point (6h and 24h) $(\mathrm{P}<0.05)$; compared with the $\mathrm{IH}+\mathrm{I} / \mathrm{R}$ group, the inhibitor group also showed enhanced expression of beclin 1 protein at each time point $(6 \mathrm{~h}$ and $24 \mathrm{~h})(\mathrm{P}<0.05)$. (See Table 3)

\section{DISCUSSION}

In the present study, we found intermittent hypoxia preconditioning can aggravate the nerve injury of the global cerebral ischemia-reperfusion model, and the mechanism is associated with the activation of mTOR/ autophagy pathway. Our results provided experimental basis for the prevention and cure of OSAHS complicated with ischemic cerebrovascular diseases.

Autophagy widely exists in eukaryotic organisms, which is a phenomenon that cells recognize, degrade and reverse dysfunctional proteins and organelles via the lysosome pathway, stimulated by physiological or pathological factors. It is the third manner of death besides necrosis and apoptosis [8-9]. The results of the study showed that compared with the $\mathrm{I} / \mathrm{R}$ group, the $\mathrm{IH}+\mathrm{I} / \mathrm{R}$ group showed aggravated structural damage of neurons and a decreased survival density of neurons at each time point (6h and 24h) as well as enhanced expression of beclin1 protein detected by immunohistochemical staining and RTPCR, which indicated that autophagy exists in the process of pure cerebral ischemia-reperfusion, and intermittent hypoxia can further activate autophagy and worsen neuron 
Table 1: Intergroup comparison of the survival rates of hippocampus neurons $(\mathrm{n}=5, \%)$

\begin{tabular}{lccc}
\hline GROUPS & n & \multicolumn{2}{c}{ Survival rate of neuron } \\
\cline { 3 - 4 } & & $\mathbf{6 h}$ & $\mathbf{2 4 h}$ \\
\hline SO group & 5 & $99.08 \pm 0.84$ & $98.65 \pm 0.75$ \\
I/R group & 5 & $78.65 \pm 1.43 *$ & $71.71 \pm 1.39 *$ \\
IH+I/R group & 5 & $49.06 \pm 1.70 * \triangle$ & $45.12 \pm 1.51 * \triangle$ \\
Inhibitor group & 5 & $55.03 \pm 1.63 * \triangle \boldsymbol{5}$ & $50.32 \pm 1.42 * \triangle \boldsymbol{\Delta}$ \\
\hline
\end{tabular}

Note: $* P<0.05$, compared with the $\mathrm{SO}$ group; $\triangle P<0.05$, compared with the $\mathrm{I} / \mathrm{R}$ group; $\wedge P<0.05$, compared with the IH+I/R group

loss after cerebral ischemia. These findings are consistent with existed research results at home and abroad. Liu [10] built the intermittent hypoxia mouse model and observed the expression of autophagy-associated proteins of the nerve cells in the mouse hippocampus CA1 region after intermittent hypoxia, and the results showed an increased level of LC3II/LC3 $\equiv$ protein and exacerbated ultrastructural
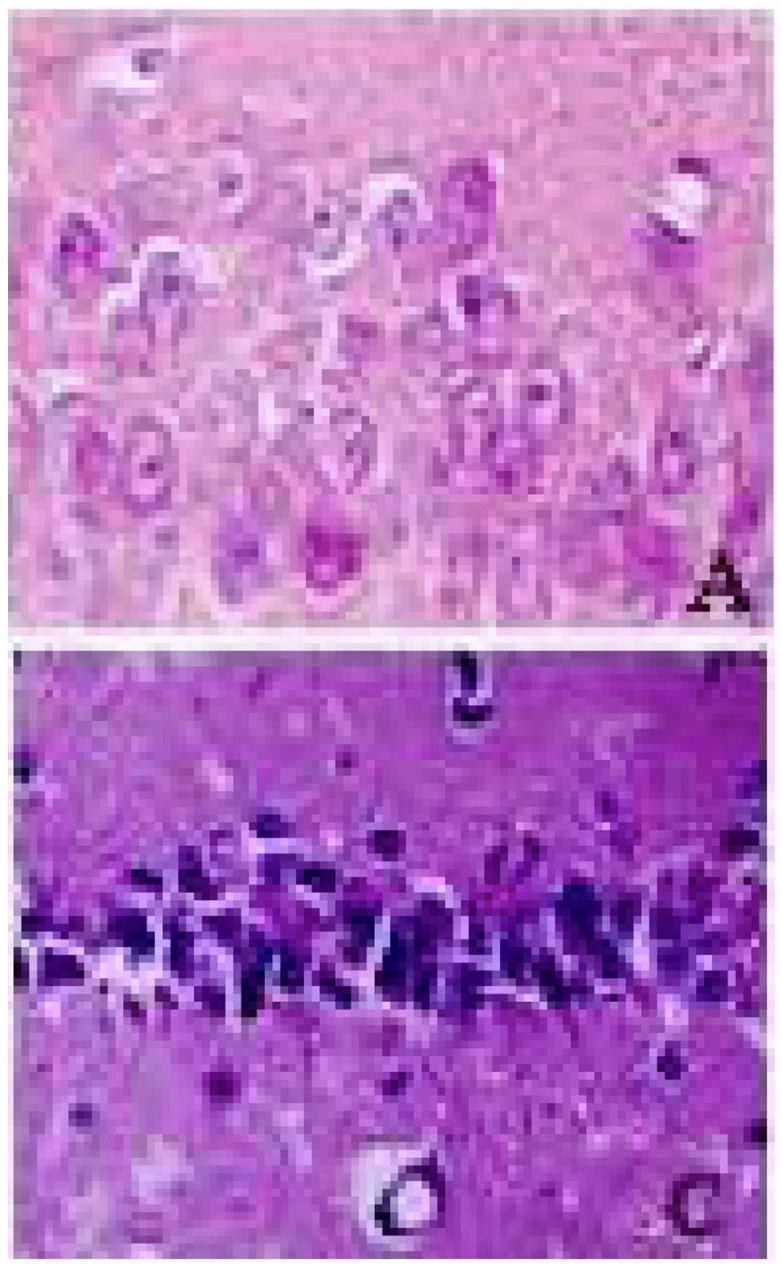

damage of neurons, which indicated that intermittent hypoxia could induce autophagy in nerve cells of the rat hippocampus. Koike et al. [11] found in the ischemia hypoxia mouse model a remarkable rise of the level of LC3-II protein, the formation of autophagosomes in the pyramidal cell layer of the hippocampus and extensive death of hippocampal neurons, which suggested that the
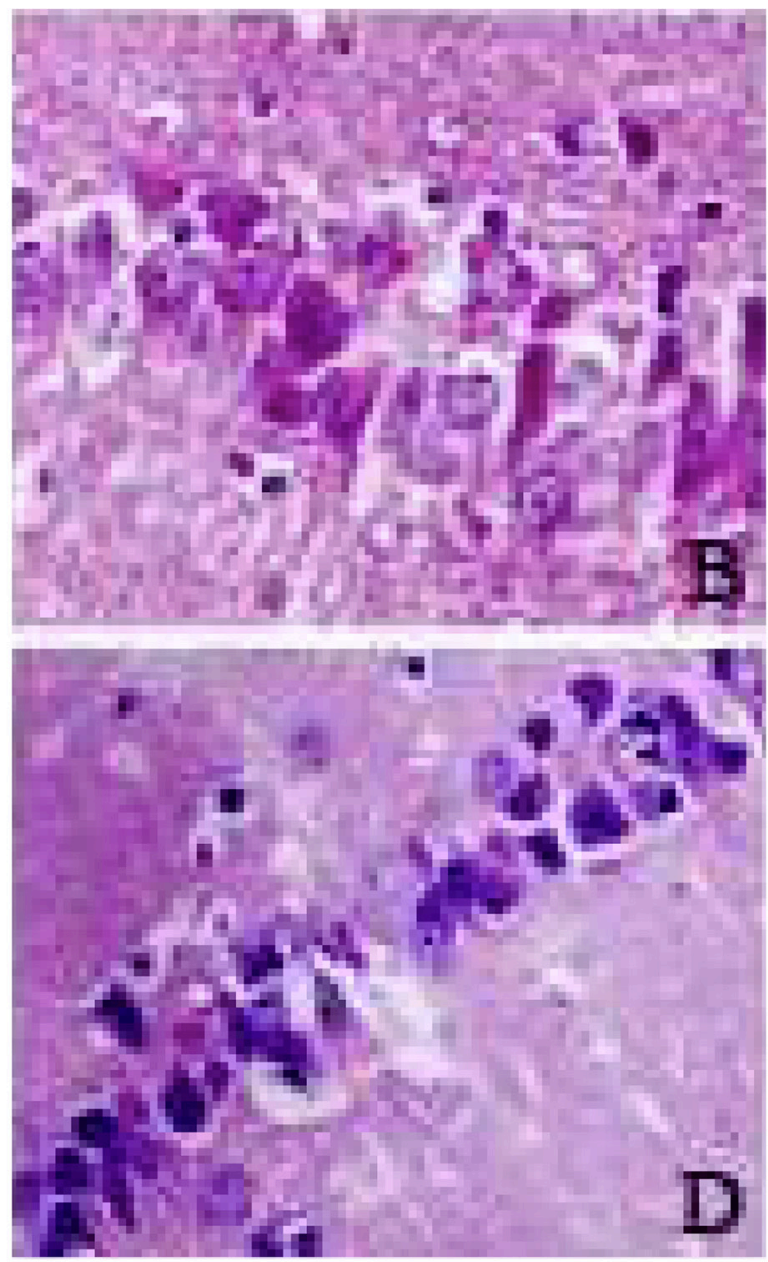

Figure 1: Hippocampus neurons of each group 24h after cerebral ischemia (HE staining, 400x). A. SO group; B. I/R group; C. $\mathrm{IH}+\mathrm{I} / \mathrm{R}$ group; D. inhibitor group. 
Table 2: Intergroup comparison of the counts of mTOR or beclin1 immune-positive cells in the hippocampus $( \pm s$, cells/ visual field at high magnification)

\begin{tabular}{|c|c|c|c|c|c|}
\hline \multirow[t]{2}{*}{ Groups } & \multirow[t]{2}{*}{$\mathbf{n}$} & \multicolumn{2}{|c|}{ mTOR } & \multicolumn{2}{|c|}{ beclin1 } \\
\hline & & $6 \mathrm{~h}$ & $24 \mathrm{~h}$ & $6 \mathrm{~h}$ & $24 \mathrm{~h}$ \\
\hline SO group & 5 & $14.65 \pm 0.48$ & $15.40 \pm 0.58$ & $2.06 \pm 0.23$ & $2.10 \pm 0.30$ \\
\hline I/R group & 5 & $22.38 \pm 0.46^{*}$ & $24.16 \pm 0.60^{*}$ & $8.58 \pm 0.58 *$ & $10.58 \pm 0.49^{*}$ \\
\hline $\mathrm{IH}+\mathrm{I} / \mathrm{R}$ group & 5 & $30.40 \pm 0.43 * \triangle$ & $32.86 \pm 0.50 * \triangle$ & $15.57 \pm 0.57 * \triangle$ & $18.78 \pm 0.43 * \triangle$ \\
\hline Inhibitor group & 5 & $26.60 \pm 0.37 * \triangle \Delta$ & $28.51 \pm 0.52 * \triangle \Delta$ & $21.74 \pm 0.51 * \triangle \Delta$ & $24.32 \pm 0.49 * \triangle \Delta$ \\
\hline
\end{tabular}

Note: $* P<0.05$, compared with the SO group; $\triangle P<0.05$, compared with the $\mathrm{I} / \mathrm{R}$ group; ${ }^{\wedge} P<0.05$, compared with the IH+I/R group

activation of autophagy led to enhanced expression of LC3II and aggravated neuron injury.

mTOR signal pathway plays an important role in central nervous system diseases. For instance, $\mathrm{Su}$ [12] treated the mice suffering cerebral ischemia with rapamycin, and found that rapamycin could up-regulate the phosphorylation level of mTOR protein and reduce the cerebral infarct area, which indicated that the protective effect of a trace amount of rapamycin on acute cerebral ischemic injury was correlated with the activation of mTOR signal pathway. $\mathrm{Xu}$ [13] established the SAH model with SD rats which were treated with rapamycin, and detected the expression of phosphorylated mTOR by Western blot, and the results showed enhanced activity of mTOR after SAH, which indicated that a trace amount of rapamycin could down-regulate the phorylation level of mTOR protein and reduce the degree of edema and necrosis of brain tissue. Based on our results in this study, immunohistochemical staining and RT-PCR both showed enhanced expression of mTOR protein in the $\mathrm{IH}+\mathrm{I} / \mathrm{R}$ group at each time point (6h and 24h) compared with the I/R group, which indicated that intermittent hypoxia could activate mTOR signal pathway and deteriorate nerve injury after cerebral ischemia. Studies [14-16] showed that stress responses such as hypoxia, ischemia, low-energy, could regulate the activity of mTORC1 via up-regulating AMPK activity, changing the aggregative state of TSC1/2, phosphorylating TSC2 or directly phosphorylating raptor; and inflammatory factors such as TNF- $\alpha$ could trigger similar disaggregation of TSC1/2 through IKK $\beta$ or Wnt signal pathway so as to activate mTORC1. Therefore, intermittent hypoxia can activate mTOR signal pathway to worsen cerebral injury by aggravating anoxia, ischemia and the release of inflammatory factors such as TNF- $\alpha$ in the central nervous system after cerebral ischemia. Recent researches show that mTOR kinase is a pivotal regulatory site of autophagy, which closely correlates with the onset and development of autophagy, and inihibiting mTOR activity can up-regulate autophagy [17-18]. Tizon et al. treated the mice suffering cerebral ischemia
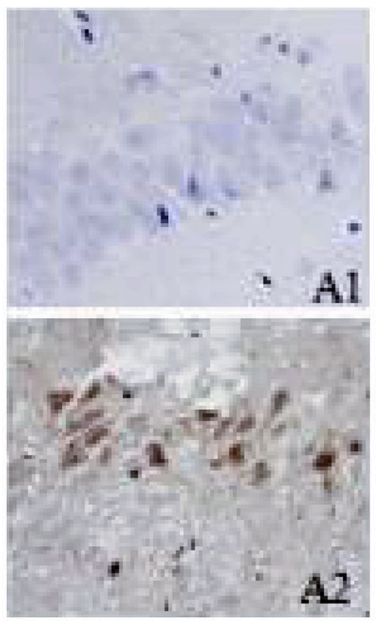
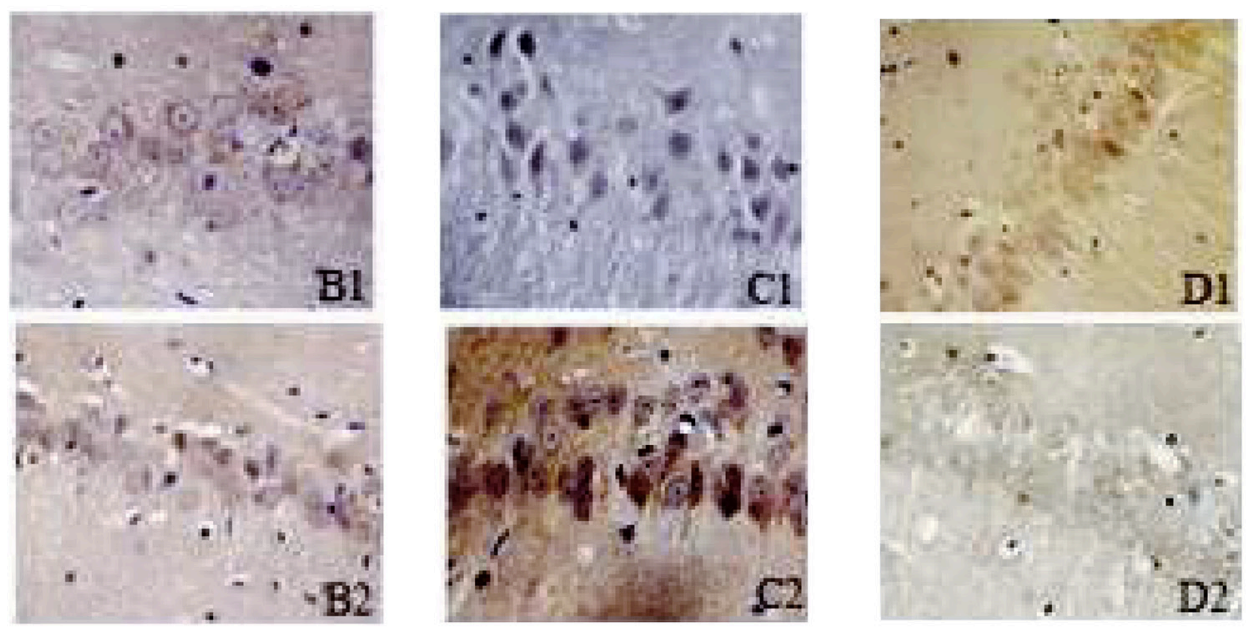

Figure 2: Positive expression of mTOR or beclin1 in the hippocampus of each group $24 \mathrm{~h}$ after cerebral ischemia (immunohistochemical staining, 400x). A1-D1: immunohistochemical results of beclin1 in the hippocampus for SO, I/R, IH+I/R and inhibitor group respectively. A2-D2: immunohistochemical results of mTOR in the hippocampus for SO, I/R, IH+I/R and inhibitor group respectively. 
Table 3: Changes of the relative expression levels of mTOR and beclin1 mRNA in the hippocampus of each group $(\bar{x} \pm s)$

\begin{tabular}{|c|c|c|c|c|c|}
\hline \multirow[t]{2}{*}{ Group } & \multirow[t]{2}{*}{$\mathbf{N}$} & \multicolumn{2}{|c|}{ mTOR } & \multicolumn{2}{|c|}{ beclin1 } \\
\hline & & $6 \mathrm{~h}$ & $24 \mathrm{~h}$ & $6 \mathrm{~h}$ & $24 \mathrm{~h}$ \\
\hline SO group & 5 & $1.00 \pm 0.00$ & $1.00 \pm 0.00$ & $1.00 \pm 0.00$ & $1.00 \pm 0.00$ \\
\hline I/R group & 5 & $1.22 \pm 0.10^{*}$ & $1.27 \pm 0.08^{*}$ & $1.57 \pm 0.11 *$ & $1.79 \pm 0.09 *$ \\
\hline $\mathrm{IH}+\mathrm{I} / \mathrm{R}$ group & 5 & $1.65 \pm 0.06 * \triangle$ & $1.73 \pm 0.07 * \triangle$ & $2.37 \pm 0.14 * \triangle$ & $2.54 \pm 0.24 * \triangle$ \\
\hline Inhibitor group & 5 & $1.32 \pm 0.07 * \triangle \Delta$ & $1.43 \pm 0.06^{* \triangle \Delta}$ & $2.87 \pm 0.10 * \triangle \Delta$ & $2.94 \pm 0.15^{*} \triangle \Delta$ \\
\hline
\end{tabular}

Note: $* P<0.05$, compared with the SO group; $\triangle P<0.05$, compared with the $\mathrm{I} / \mathrm{R}$ group; ${ }^{\wedge} P<0.05$, compared with the IH+I/R group

with cysteine protease inhibitor $\mathrm{C}$ and the results showed decreased activity of mTOR signal pathway, up-regulated autophagy and a lower degree of neuronic injury, which suggested that inhibiting mTOR pathway could induce autophagy so as to exert protective effects on cortex neurons of mice [19]. Zhu et al. [20] found that downregulating phosphorylation of mTOR via blocking P13K pathway with type IP13K kinase inhibitor could inhibit autophagy induced by high glucose and promote apoptosis induced by high glucose, which indicated that mTOR signal pathway plays an important part in autophagy induced by high glucose. Based on our results in this study, immunohistochemical staining and RT-PCR both showed down-regulated expression of mTOR protein and up-regulated expression of beclin 1 protein in the inhibitor group at each time point (6h and $24 \mathrm{~h}$ ) compared with the $\mathrm{IH}+\mathrm{I} / \mathrm{R}$ group, which indicated that $\mathrm{mTOR}$ inhibitor could suppress the expression of mTOR protein and then promote the occurrence of autophagy.

Several limitations should be mentioned. Firstly, we performed experiment only in vivo but not in vitro. Secondly, the results may be more stable if we verified the results in cell model. Finally, the interaction between these two proteins was not be analyzed.

In conclusion, intermittent hypoxia can aggravate the nerve injury of global cerebral ischemia-reperfusion via activating $\mathrm{mTOR} /$ autophagy pathway.

\section{MATERIALS AND METHODS}

\section{Materials}

Eighty male Wistar rats were purchased from Beijing Charles River Co., Ltd (license: SCXK).

\section{Grouping and model preparing}

The rats were divided into four groups by the random number method: $\mathrm{SO}$ group $(\mathrm{n}=20), \mathrm{I} / \mathrm{R}$ group $(n=20), I H 7+I / R$ group $(n=20), I H 21+I / R$ group $(n=20)$. Each group was subdivided into two time-point groups: $6 \mathrm{~h}$ group $(\mathrm{n}=10)$ and $24 \mathrm{~h}$ group $(\mathrm{n}=10)$.
In the SO group, vessels were exposed and isolated, electrocoagulation was not performed to the vertebral artery and the common carotid artery was not clipped. In the $\mathrm{I} / \mathrm{R}, \mathrm{IH} 7+\mathrm{I} / \mathrm{R}$ and $\mathrm{IH} 21+\mathrm{I} / \mathrm{R}$ group, rat models of global cerebral ischemia were performed with improved Pulsinelli four-vessel occlusion method, and the procedures were as follows: the rats received anesthesia with $10 \%$ chloral hydrate and then were fixed at prone position; the back of the occipitalia was cut open in the middle to expose the double flank holes; the preheated electrocoagulation needle was inserted (each time lasted for 2-4s) to seal the bilateral vertebral arteries; the rats were then placed on the surgery board in lying position, and the neck was cut open in the middle to isolate the bilateral common carotid arteries; twentyfour hours later, the bilateral common carotid arteries were simultaneously clipped for $15 \mathrm{~min}$ to cause global cerebral ischemia.

From 8:00am-15:00pm every day, the rats in the $\mathrm{I} / \mathrm{R}, \mathrm{IH} 7+\mathrm{I} / \mathrm{R}$ and $\mathrm{IH} 21+\mathrm{I} / \mathrm{R}$ group stayed in the hypoxic chamber where nitrogen and air alternated with one circle lasting for $120 \mathrm{~s}$. Nitrogen entry continued for $30 \mathrm{~s}$ to maintain an oxygen concentration of $5-21 \%$ internally. A digital oxygen meter was employed to monitor the internal oxygen concentration. The whole process continued for $21 \mathrm{~d}$.

\section{HE staining}

Five rats were randomly selected from each group $6 \mathrm{~h}$ and $24 \mathrm{~h}$ after cerebral ischemia-reperfusion respectively, and fixed by cardiac perfusion with $4 \%$ paraformaldehyde. Then brain was harvested after decollation to cut out brain tissue from optic chiasma to cerebral transverse fissure. After the procedures of dehydration, paraffin embedding, sectioning, deparaffinating, xylene clearing and $\mathrm{HE}$ staining were performed routinely, the sections were observed under the optical microscope (40X10).

\section{Immunohistochemical staining}

The above-mentioned brain tissue sections were routinely deparaffinated, blocked with $0.3 \% \mathrm{H}_{2} \mathrm{O}_{2}$ for 
$10 \mathrm{~min}$ and subjected to heat induced epitope retrieval for $1.5 \mathrm{~min}$. After anti-beclin 1 and anti-mTOR antibody was added, the sections were incubated in the wet box at $37^{\circ} \mathrm{C}$ for $30 \mathrm{~min}$. Then secondary antibody was added, followed by incubation in the $37^{\circ} \mathrm{C}$ incubator for another $40 \mathrm{~min}$. Next, after DAB development, dehydration, clearing and sealing with neutral balsam, the sections were observed under the microscope. Quantitative analysis of positive rates: five sections were selected for analyzing each index, and five view fields were randomly selected in the hippocampus on each section under the optic microscope (400X), and positive cells of each view field were counted with the computer.

\section{RT-PCR}

Five rats were randomly selected from each group $6 \mathrm{~h}$ and $24 \mathrm{~h}$ after cerebral ischemia-reperfusion respectively. Tissue $(50 \sim 100 \mathrm{mg})$ was collected from the hippocampus CA1 region of the rats and then well grinded in $1 \mathrm{~mL}$ Trizol reagent to extract total RNA. OD260/280 values were measured with Rotor-Gene3000 fluorogenic quantitative PCR amplifier, and RNA concentrations were calculated with OD260 values. RNA was then stored at $-80^{\circ} \mathrm{C}$. Detection procedures: the forward and reverse primers of mTOR were GGTGGACGAGCTCTTTGTCA and AGGAGCCCTAACACTCGGAT; the forward and reverse primers of beclin1 were CTCTCGT CAAGGCGTCACTTC and CCTTAGACCCCTCCATT CCTCA; RT-PCR reactions: Stage 1, 2 (reverse transcription reactions): Reps: $1,42^{\circ} \mathrm{C} 50 \mathrm{~min}, 95^{\circ} \mathrm{C} 10 \mathrm{sec}$; Stage 3 (PCR reactions): Reps: $45,95^{\circ} \mathrm{C} 15 \mathrm{~s}, 56^{\circ} \mathrm{C}$ 20s; Stage4 (dissociation curve analysis): Dissociation Protocol.

\section{Statistical analysis}

A database of the data obtained in the study was established using Excel 2003 software. All data were shown with $\bar{x} \pm \mathrm{s}$. One-way analysis of variance for repeated data was performed with SPSS17.0 software. $\mathrm{P}<0.05$ indicated significant difference.

\section{CONFLICTS OF INTEREST}

We state that we have no conflicts to disclose.

\section{REFERENCES}

1. Deng XZ, Liu B and Li Y. Research Progress of Obstructive Sleep Apnea Hyponea Syndrome and Hypertension. Advances in Cardiovascular Diseases 2014; 35: 230-233.

2. Cho ER, Kim H, Seo HS, Suh S, Lee SK and Shin C. Obstructive sleep apnea as a risk factor for silent cerebral infarction. J Sleep Res 2013; 22: 452- 458.
3. Wang HY and Xu E. Dual roles of autophagy in cerebral ischemia. International Journal of Cerebrovascular Diseases 2012; 20: 237-240.

4. Liu L, Fang YQ, Xue ZF, He YP, Fang RM and Li L. Betaasarone attenuates ischemia-reperfusion-induced autophagy in rat brains via modulating JNK, p-JNK, Bcl-2 and Beclin 1. Eur J Pharmacol 2012; 680: 34-40.

5. Sengupta S, Peterson TR and Sabatini DM. Regulation of the mTOR complex 1 pathway by nutrients, growth factors, and stress. Mol Cell 2010; 40: 310-322.

6. Lin Z, McDermott A, Shao L, Kannan A, Morgan M, Stack BC Jr, Moreno M, Davis DA, Cornelius LA and Gao L. Chronic mTOR activation promotes cell survival in Merkel cell carcinoma. Cancer Lett 2014; 344: 272-281.

7. Cao ZB. MTOR signaling pathway in the role and mechanism of brain ischemia. Zheng Zou Da Xue 2012;

8. Ding H, Tang YH and Huang XP. The role of autophagy in cerebral ischemic injury. Chin Pharm Bull 2015; 31: 1048-1052.

9. Ropolo A, Bagnes CI, Molejon MI, Lo Re A, Boggio V, Gonzalez CD and Vaccaro MI. Chemotherapy and autophagy-mediated cell death in pancreatic cancer cells. Pancreatology 2012; 12: 1-7.

10. Liu HY, Chen R, Li YN, Zhang YL and Liu CF. Chronic intermittent hypoxia area in mice hippocampal CAl neurons autophagy. Chin J Tuberc Respir Dis.2011; 34: 467-469.

11. Koike M, Shibata M, Tadakoshi M, Gotoh K, Komatsu M, Waguri S, Kawahara N, Kuida K, Nagata S, Kominami E, Tanaka K and Uchiyama Y. Inhibition of autophagy prevents hippocampal pyramidal neuron death after hypoxic-ischemic injury. Am J Pathol 2008; 172: 454-469.

12. Su FY. Trace rapamycin to study the protective effect and mechanism of ischemic brain damage. Second Military Medical University 2011.

13. Xu X. MTOR signaling pathway in the role of early brain injury after subarachnoid hemorrhage and mechanism research. Su Zhou Da Xue 2014;

14. He JY and Liu F. MTOR signaling pathway major diseases associated with aging and aging. Progress in Biochemistry and Biophysics 2014; 41: 257-265.

15. Lee DF, Kuo HP, Chen CT, Hsu JM, Chou CK, Wei Y, Sun HL, Li LY, Ping B, Huang WC, He X, Hung JY, Lai CC, Ding Q, Su JL, Yang JY, Sahin AA, Hortobagyi GN, Tsai FJ, Tsai $\mathrm{CH}$ and Hung MC. IKK beta suppression of TSC1 links inflammation and tumor angiogenesis via the mTOR pathway. Cell 2007; 130: 440-455.

16. Thomas GV, Tran C, Mellinghoff IK, Welsbie DS, Chan E, Fueger B, Czernin J and Sawyers CL. Hypoxiainducible factor determines sensitivity to inhibitors of mTOR in kidney cancer. Nat Med 2006; 12: 122-127.

17. Singh BN, Kumar D, Shankar S and Srivastava RK. Rottlerin induces autophagy which leads to apoptotic cell death through inhibition of PI3K /Akt/mTOR pathway in human pancreatic cancer stem cells. Biochem Pharmacol 2012; 84: 1154-1163. 
18. Zhao MM, Zhao YB, Luo P, Li SZ, Xu HX, Huo K, Zhang L, Liu WB, Wu Y, Jiang XF, Yue F, Liu ZB and Fei Z. PI3K/ Akt signal pathway of autophagy induced by mechanical damage of neurons. The neurosurgical disease research magazine 2012; 11: 491-494.

19. Tizon B, Sahoo S, Yu H, Gauthier S, Kumar AR, Mohan P, Figliola M, Pawlik M, Grubb A, Uchiyama Y, Bandyopadhyay U, Cuervo AM, Nixon RA, et al. Induction of autophagy by cystatin $\mathrm{C}$ : a mechanism that protects murine primary cortical neurons add neuronal cell lines. PLOS One 2010; 5: 9819.

20. Zhu JL, Ma TA, Chen XH, Yang Q and Ding GH. High glucose increases podocyte autophagy through PI3K-AKTmTOR signaling pathway. Chinese Journal of Nephrology 2013; 29: 515-519. 